\title{
Características clínicas, eléctricas y ecocardiográficas de los pacientes con bloqueo interauricular avanzado
}

\author{
Clinical, electrical and echocardiographic characteristics of patients with advanced \\ interatrial block \\ Delicia Gentille-Lorente ${ }^{1 *}$ y Teresa Salvadó-Usach ${ }^{2}$ \\ ${ }^{1}$ Servicio de Cardiología; 2 Servicio de Anatomía Patológica, Hospital de Tortosa Verge de la Cinta IISPV. Tortosa, Tarragona, España
}

El haz de Bachmann se integra con un conjunto de fibras miocárdicas paralelas especializadas y representa el 80 a $85 \%$ de la conducción interauricular. Discurre por las paredes anterosuperiores auriculares y su afectación da lugar al bloqueo interauricular (BIA), que es: a) parcial (BIA-p) si la conducción está retrasada (en el ECG produce una onda $P \geq 120 \mathrm{~ms}$ ) o b) avanzado (BIA-a) si está del todo interrumpida y la despolarización auricular izquierda (Al) ocurre en dirección retrógrada caudocraneal (la onda $P$ es $\geq 120$ ms y bifásica \pm en las derivaciones inferiores II, III y $V F)^{1}$. El BIA-a produce una disfunción eléctrica y mecánica auricular que propicia el desarrollo de una "miopatía fibrótica auricular"2,3; la fibrosis favorece la estasis sanguínea y la trombosis intrauricular (en especial en la orejuela) ${ }^{4}$, así como el desarrollo de arritmias supraventriculares como el flúter (típico y atípico), y sobre todo la fibrilación auricular (FA): el denominado síndrome de Bayés $^{3-8}$. Existe evidencia de que el BIA-a es un marcador de disfunción morfofuncional auricular y un predictor de episodios como FA o flúter (de novo o recidivas tras cardioversión o ablación), accidente cerebral vascular (ACV) embólico e insuficiencia cardíaca $^{3-10}$. La patogenia del BIA no se conoce por completo, pero en su génesis intervienen factores degenerativos, isquémicos, infiltrativos e inflamatorios (la edad es el factor más determinante); en consecuencia, puede aparecer exista o no una cardiopatía subyacente, y cualquiera que sea el tamaño Al, aunque por lo general ocurre en las Al dilatadas; puede ser intermitente y dependiente de la frecuencia (BIA de segundo grado) e incluso reversible, así como la remodelación eléctrica auricular. La prevalencia del BIA aumenta de forma exponencial con la edad y es alta en la población general (59\% en los mayores de 60 años) y en diferentes poblaciones de pacientes (41-48\%).

Con la finalidad de caracterizar clínica, eléctrica y ecocardiográficamente a los pacientes con BIA, y analizar su relación con la FA/flúter y el ACV, se llevó a cabo este estudio. Se diseñó un protocolo descriptivo, observacional y retrospectivo de pacientes consecutivos, mayores de edad, visitados en una consulta de alta resolución de cardiología (visita con electrocardiograma y ecocardiograma) durante tres años; todos ellos se hallaban en ritmo sinusal durante la visita. Se reconocieron 63 casos de BIA-a en el ECG y por tanto se seleccionó una muestra similar de pacientes consecutivos con BIA-p (Fig. 1) y sin BIA que conformó una muestra total de 165 pacientes para el estudio. Las variables analizadas se obtuvieron de la historia clínica informatizada y de la visita realizada (parámetros 
Tabla 1. Características de los pacientes sin BIA, con BIA parcial y con BIA avanzado

\begin{tabular}{|c|c|c|c|c|}
\hline & $\begin{array}{l}\text { P normal } \\
(n=52)\end{array}$ & $\begin{array}{l}\text { BIA parcial } \\
(n=50)\end{array}$ & $\begin{array}{c}\text { BIA avanzado } \\
\quad(n=63)\end{array}$ & p \\
\hline Edad (años), $\bar{X} \pm D E$ & $59.8 \pm 13.3$ & $66.9 \pm 11.4$ & $76.4 \pm 8,2$ & $<0.0001$ \\
\hline Edad $\geq 65$ años, n (\%) & $22(42.3)$ & $33(66.0)$ & $57(90.5)$ & $<0.0001$ \\
\hline Sexo varón, n (\%) & $25(48.1)$ & $32(64.0)$ & $41(65.1)$ & 0.132 \\
\hline $\begin{array}{l}\text { Antecedentes clínicos } \\
\text { Hipertensión arterial, } \mathrm{n}(\%) \\
\text { Diabetes mellitus, } \mathrm{n}(\%) \\
\text { Dislipemia, } \mathrm{n}(\%) \\
\text { Tabaquismo y ex tabaquismo, } \mathrm{n}(\%) \\
\text { Índice de masa corporal }\left(\mathrm{kg} / \mathrm{m}^{2}\right), \overline{\mathrm{X}} \pm \mathrm{DE} \\
\left.\text { Obesidad (IMC } \geq 30 \mathrm{~kg} / \mathrm{m}^{2}\right), \mathrm{n}(\%) \\
\text { Filtración glomerular }(\mathrm{ml} / \mathrm{min}), \overline{\mathrm{X}} \pm \mathrm{DE} \\
\text { Enfermedad renal crónica estadio } \geq 2(\mathrm{FG}<90 \mathrm{ml} / \mathrm{min}), \mathrm{n}(\%) \\
\text { Enfermedad renal crónica estadio } \geq 3(\mathrm{FG}<60 \mathrm{ml} / \mathrm{min}), \mathrm{n}(\%) \\
\text { EPOC, } \mathrm{n}(\%) \\
\text { SAHOS confirmado o roncador }+ \text { apneas } \\
\text { Cardiopatía isquémica, } \mathrm{n}(\%) \\
\text { FA/flúter, } \mathrm{n}(\%) \\
\text { Accidente cerebral vascular, } \mathrm{n}(\%) \\
\text { Ictus, } \mathrm{n}(\%) \\
\text { Accidente isquémico transitorio, } \mathrm{n}(\%)\end{array}$ & $\begin{array}{c}28(53.8) \\
7(13.5) \\
31(59.6) \\
19(36.5) \\
27.9 \pm 4.7 \\
18(34.6) \\
88.7 \pm 23.3 \\
22(43.1) \\
4(7.7) \\
5(9.6) \\
8(15.4) \\
5(9.6) \\
22(42.3) \\
0(0.0) \\
0(0.0) \\
0(0.0)\end{array}$ & $\begin{array}{c}33(66.0) \\
5(10.0) \\
33(66.0) \\
24(48.0) \\
29.0 \pm 4.3 \\
21(42.0) \\
79.2 \pm 19.1 \\
34(68.0) \\
6(12.0) \\
2(4.0) \\
6(12.0) \\
10(20.4) \\
15(30.0) \\
1(2.0) \\
1(2.0) \\
0(0.0)\end{array}$ & $\begin{array}{c}55(87.3) \\
24(38.1) \\
47(74.6) \\
21(33.3) \\
30.2 \pm 5.2 \\
31(52.5) \\
70.5 \pm 23.2 \\
50(79.4) \\
20(32.3) \\
9(14.3) \\
8(13.6) \\
14(22.2) \\
17(27.0) \\
10(15.9) \\
8(12.7) \\
2(3.2)\end{array}$ & $\begin{array}{c}<0.0001 \\
<0.0001 \\
0.228 \\
0.261 \\
0.045 \\
0.159 \\
<0.0001 \\
<0.0001 \\
0.001 \\
0.186 \\
0.883 \\
0.177 \\
0.194 \\
\text { na } \\
\text { na } \\
\text { na }\end{array}$ \\
\hline $\begin{array}{l}\text { Electrocardiograma } \\
\text { Bloqueo auriculoventricular, n (\%) } \\
\text { Bloqueo de rama del haz de His completo, n (\%) } \\
\text { Bloqueo del fascículo anterior o posterior, n (\%) } \\
\text { Índice de Cornell positivo, n (\%) } \\
\text { Índice de Sokolow-Lyon positivo, n (\%) }\end{array}$ & $\begin{array}{l}2(3.8) \\
3(5.8) \\
5(9.6) \\
2(3.8) \\
1(1.9)\end{array}$ & $\begin{array}{c}3(6.0) \\
10(20.0) \\
9(18.0) \\
6(12.0) \\
3(6.0)\end{array}$ & $\begin{array}{l}20(31.7) \\
13(20.6) \\
18(29.5) \\
8(12.7) \\
4(6.3)\end{array}$ & $\begin{array}{c}<00001 \\
0.057 \\
0.225 \\
0.225 \\
0.493\end{array}$ \\
\hline $\begin{array}{l}\text { Ecocardiograma } \\
\text { Hipertrofia } \mathrm{VI}, \mathrm{n}(\%) \\
\text { Tabique interventricular sigmoideo ( } \geq 13 \mathrm{~mm}), \mathrm{n}(\%) \\
\text { Tabique interventricular sigmoideo, } \overline{\mathrm{X}} \pm \mathrm{DE} \\
\text { Fracción de expulsión del } \mathrm{VI}, \overline{\mathrm{X}}_{ \pm} \mathrm{DE} \\
\text { Fracción de expulsión del } \mathrm{VI} \leq 51 \%, \mathrm{n}(\%) \\
\text { Fracción de expulsión del } \mathrm{VI}<40 \%, \mathrm{n}(\%) \\
\text { Disfunción diastólica, } \mathrm{n}(\%)\end{array}$ & $\begin{array}{l}7(13.7) \\
7(13.5) \\
13.6 \pm 2.1 \\
61.75 \pm 7.06 \\
2(3.8) \\
0(0.0) \\
15(30.0)\end{array}$ & $\begin{array}{c}10(20.0) \\
10(20.0) \\
15.0 \pm 2.6 \\
57.5 \pm 2.2 \\
11(22.0) \\
3(6.0) \\
19(40.4)\end{array}$ & $\begin{array}{c}16(28.6) \\
16(25.4) \\
16.3 \pm 2.5 \\
60.7 \pm 11.1 \\
7(12.5) \\
4(7.1) \\
38(71.7)\end{array}$ & $\begin{array}{l}0.166 \\
0.281 \\
0.058 \\
0.103 \\
0.022 \\
0.159 \\
<00001\end{array}$ \\
\hline $\begin{array}{l}\text { Disfunción diastólica, n (\%) } \\
\text { No } \\
\text { Ligera } \\
\geq \text { moderada }\end{array}$ & $\begin{array}{c}35(70.0) \\
11(22.0) \\
4(8.0)\end{array}$ & $\begin{array}{c}28(59.6) \\
14(29.8) \\
5(10.6)\end{array}$ & $\begin{array}{l}15(28.3) \\
25(47.2) \\
13(24.5)\end{array}$ & $<00001$ \\
\hline Área $4 \mathrm{C} \mathrm{Al}\left(\mathrm{cm}^{2}\right), \overline{\mathrm{X}} \pm \mathrm{DE}$ & $18.6 \pm 4.0$ & $21.5 \pm 4.9$ & $23.3 \pm 4.7$ & $<00001$ \\
\hline Volumen biplano indexado $\mathrm{Al}\left(\mathrm{ml} / \mathrm{cm}^{2}\right), \bar{X} \pm \mathrm{DE}$ & $30.0 \pm 8.5$ & $37.8 \pm 11.0$ & $39.5 \pm 11.8$ & 0.002 \\
\hline Al dilatada, $\mathrm{n}(\%)$ & $16(30.8)$ & $33(66.0)$ & $44(78.6)$ & $<00001$ \\
\hline Calcificación del anillo mitral, n (\%) & $3(5.8)$ & $3(6.0)$ & $2(21.8)$ & 0.011 \\
\hline Valvulopatía izquierda $\geq$ moderada, n (\%) & $8(15.4)$ & $10(20.0)$ & $12(19.0)$ & 0.812 \\
\hline Valvulopatía mitral relevante (insuficiencia $\geq$ moderada 0 est & $5(9.6)$ & $6(12.0)$ & $7(11.1)$ & 0.926 \\
\hline
\end{tabular}

Al: aurícula izquierda; BIA: bloqueo interauricular; EPOC: enfermedad pulmonar obstructiva crónica; na: no aplicable; SAHOS: Síndrome de apnea obstructiva del sueño; VI: ventrículo izquierdo

electrocardiográficos y ecocardiográficos). Mediante SPSS 19.0 se efectuaron un estudio descriptivo (que expresó los resultados de las variables cuantitativas como media y desviación estándar y los de las cualitativas como porcentaje) y un análisis bivariante (mediante las pruebas ji cuadrada, exacta de Fisher, $\mathrm{t}$ de Student y $U$ de Mann-Whitney) y multivariante; el nivel de significación establecido fue $p<0.05$. 


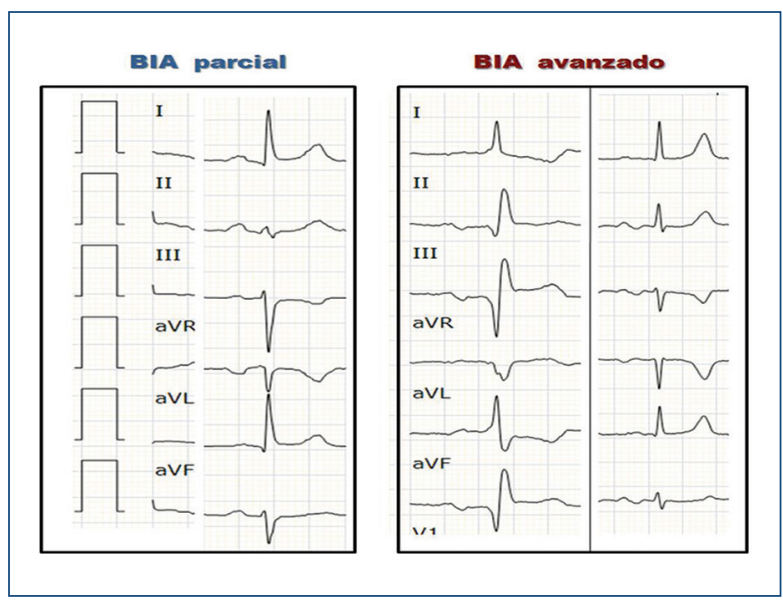

Figura 1. Panel de la izquierda: derivaciones horizontales del ECG de un paciente con BIA parcial. Panel de la derecha: derivaciones horizontales de los ECG de dos pacientes con BIA avanzado.

Tabla 2. Variables relacionadas con riesgo de presentar un BIA avanzado

\begin{tabular}{|l|c|c|c|}
\hline & $\mathbf{O R}$ & IC 95\% & P \\
\hline Edad $\geq 65$ años & 6.1 & $1.6-23.7$ & 0.009 \\
\hline Diabetes mellitus & 6.1 & $2.1-17.8$ & 0.001 \\
\hline Dilatación de la Al & 6.0 & $2.0-17.4$ & 0.001 \\
\hline Disfunción diastólica & 3.1 & $1.3-7.7$ & 0.013 \\
\hline
\end{tabular}

Al: aurícula izquierda; BIA: bloqueo interauricular; OR: odds ratio; IC: intervalo de confianza.

El análisis bivariado entre los tres grupos de pacientes arrojó los resultados que se muestran en la tabla 1. Destaca la sólida relación detectada entre el BIA y la edad, la hipertensión arterial, la diabetes mellitus, la enfermedad renal crónica, el bloqueo auriculoventricular de primer grado, la disfunción diastólica (en particular si conllevaba elevación de la presión intrauricular) y la dilatación de la Al (fue más frecuente la presencia de BIA-a en los pacientes con aurículas más grandes). Sin embargo, no se demostró la relación del BIA-a con el antecedente de FA/flúter "documentados", probablemente por la dificultad diagnóstica que suponen estas arritmias si no son permanentes (aunque la mayor parte de las FA persistentes, que representaban el $55 \%$ de todas ellas, y el $100 \%$ de los flúteres pertenecieron al grupo BIA-a). Destaca también que el $90.9 \%$ (10 casos) de los ACV isquémicos en la serie (cardioembólicos o de causa desconocida) ocurrieron en pacientes que presentaban un BIA-a, y apenas un $9.1 \%$ de los que tenían un BIA-p; de esos 10 casos del grupo BIA-a, el $80 \%$ correspondió a ictus y el $20 \%$ a accidentes isquémicos transitorios y, de todos ellos, sólo un $40 \%$ contaba con el antecedente de FA o flúter documentados. Al comparar a los grupos con BIA-p y BIA-a mediante análisis bivariado se detectó que sólo el BIA-a se relacionaba con presentar un ACV ( $p=0.022)$, en especial un ictus $(p=0.04)$.

Por último, se realizó un análisis multivariado (incluidos los tres grupos de pacientes y todas las variables de la tabla 1 con nivel de $p<0.1$ ), que determinó que las únicas variables relacionadas con riesgo de presentar un BIA-a eran tener una edad $\geq 65$ años (odds ratio $[\mathrm{OR}]=6.1)$, diabetes mellitus $(\mathrm{OR}=6.1)$, disfunción diastólica $(\mathrm{OR}=6.0)$ y dilatación auricular izquierda $(\mathrm{OR}=3.1)$ (Tabla 2).

Los resultados obtenidos llevan a concluir con que los factores vinculados con riesgo de padecer un BIA-a son tener $\geq 65$ años, diabetes, disfunción diastólica ventricular y aurícula izquierda dilatada. Los pacientes con BIA-a poseen una mayor tasa de ACV isquémico, sobre todo ictus, que aparece junto con antecedente de FA o sin ella (ya sea por el infradiagnóstico de la arritmia o por la miopatía auricular subyacente). Estos resultados destacan la posibilidad de realizar cribado y prevención de la FA y la necesidad de precisar si los enfermos con BIA-a deben anticoagularse a fin de prevenir en particular los ACV.

\section{Financiamiento}

Ninguno.

\section{Conflicto de intereses}

Los autores declaran no tener ningún conflicto de intereses.

\section{Responsabilidades éticas}

Protección de personas y animales. Los autores declaran que para esta investigación no se han realizado experimentos en seres humanos ni en animales.

Confidencialidad de los datos. Los autores declaran que en este artículo no aparecen datos de pacientes.

Derecho a la privacidad y consentimiento informado. Los autores declaran que en este artículo no aparecen datos de pacientes. 


\section{Bibliografía}

1. Bayés de Luna A, Platonov P, Cosío FG, Cygankiewicz I, Pastore C, Baranowski R. Interatrial blocks. A separate entity from left atrial enlargement: a consensus report. J Electrocardiol. 2012;45:445-51.

2. Hirsh B, Copeland-Halperin R, Halperin J. Fibrotic atrial cardiomyopathy, atrial fibrillation, and thromboembolism: mechanistic links and clinical inferences. J Am Coll Cadiol. 2015;65(20):2239-225.

3. Baranchuk A. Interatrial block and supraventricular arrhythmias. Clinical implications of Bayés' syndrome. Cardiotext. Minneapolis, Minnesota, USA, 2017.

4. Martínez-Selles M, Fernández Lozano I, Baranchuck A, Bayés-Genis A, Bayés de Luna A. Debemos anticoagular a pacientes en alto riesgo de sufrir fibrilación auricular? Rev Esp Cardiol. 2016;69:374-76.

5. Conde D, Baranchuk A. Bloqueo interauricular como sustrato anatómico-eléctrico de arritmias supraventriculares: síndrome de Bayés. Arch Cardiol Mex. 2014;84:32-40.
6. Baranchuk A, Enríquez A, Antiperovitch P, Alexander B, Cinier G. Advanced interatrial block as a key marker for atrial fibrillation recurrence: Bayés' syndrome. J Geriatr Cardiol. 2017;14:169-73.

7. Conde D, Seoane L, Gysel M, Mitrione S, Bayés de Luna A, Baranchuk A. Bayés' syndrome: the association between interatrial block and supraventricular arrhythmias. Expert Rev Cardiovasc Ther. 2015;13:541-50.

8. Seoane L, Cortés M, Conde D. Update on Bayés' syndrome: the association between an interatrial block and supraventricular arrhythmias. Expert Review of Cardiovascular Therapy. 2019. DOI: 10.1080/14779072.2019. 1577137.

9. Escobar-Robledo LA, Bayés de Luna A, Lupón J, Baranchuk A, Moliner P, Martínez- Sellés M, et al. Advanced interatrial block predicts new-onset atrial fibrillation and ischemic stroke in patients with heart failure: the "Bayes syndrome-HF" study. Int J Cardiol. 2018:15;271:174-80.

10. Arauz A, Arteaga C, Zapata-Gómez C, Ramos-Ventura C, Méndez B, Otiniano-Sifuentes R, et al. Infarto cerebral embólico de origen no determinado: más allá de la fibrilación auricular. 2019. DOI: 10.1016/j. nrl.2019.03 